\title{
Geometry dependence of RMT-based methods to extract the low-energy constants $\Sigma$ and $F$
}

\section{Christoph Lehner, ${ }^{* a, b}$ Shoji Hashimoto, ${ }^{c}$ and Tilo Wettig ${ }^{a}$}

${ }^{a}$ Institute for Theoretical Physics, University of Regensburg, 93040 Regensburg, Germany

${ }^{b}$ RIKEN/BNL Research Center, Brookhaven National Laboratory, Upton, NY-11973, USA

${ }^{c}$ High Energy Accelerator Research Organization (KEK), Tsukuba 305-0801, Japan

Email: clehner@quark.phy.bnl.gov, shoji.hashimoto@kek.jp,

tilo.wettig@physik.uni-regensburg.de

\begin{abstract}
We discuss how the choice of lattice geometry affects methods to extract the lowest-order lowenergy constants $\Sigma$ and $F$ of chiral pertubation theory that are based on the equivalence of random matrix theory (RMT) and QCD in the epsilon regime. We show how to minimize systematic deviations from RMT by an optimal choice of lattice geometry in the case of two light quark flavors. Finally, we determine the low-energy constants $\Sigma$ and $F$ from configurations of JLQCD with two dynamical overlap fermions.
\end{abstract}

The XXVIII International Symposium on Lattice Field Theory, Lattice2010

June 14-19, 2010

Villasimius, Italy

\footnotetext{
* Speaker.
} 


\section{Introduction}

It is well known that QCD in a finite volume $V$ at small quark masses $m$ simplifies as the Compton wavelength of the pion, $m_{\pi}^{-1}$, becomes large compared to $V^{1 / 4}$ [1]. As the theory is dominated by the constant mode of the pions, the spacetime dependence is suppressed and the low-lying eigenvalues of the Dirac operator are described by random matrix theory [2], see Ref. [3] for a review. The distribution of the smallest Dirac eigenvalues can be calculated in RMT, whereupon the low-energy constants (LECs) of chiral perturbation theory are used to map between the dimensionful quantities of QCD and the dimensionless quantities of RMT, see, e.g., Ref. [4]. These relations then allow for an alternative determination of the phenomenologically important LECs.

The lowest-order LECs are $\Sigma$ and $F$. While $\Sigma$ can be determined rather easily from the distribution of the small Dirac eigenvalues, $F$ can be determined only if one includes a suitable constant background gauge field such as isospin imaginary chemical potential $[5,6]$. In the following we discuss the geometry dependence of these methods and show how to minimize systematic deviations from RMT by an optimal choice of lattice geometry. We also compare our findings with lattice data of the two-flavor epsilon-regime run of JLQCD [7] and extract $\Sigma$ and $F$ from these configurations.

\section{The epsilon expansion at NNLO}

In this section we briefly review the epsilon expansion at next-to-next-to-leading order (NNLO) with a small imaginary chemical potential $i \mu$, see Ref. [8]. In the domain where the Compton wavelength of the pion becomes large compared to $V^{1 / 4}$, chiral perturbation theory $(\chi \mathrm{PT})$ can be reordered according to the power counting [1]

$$
V \sim \varepsilon^{-4}, \quad \partial_{\rho} \sim \varepsilon, \quad \pi(x) \sim \varepsilon, \quad m_{\pi} \sim \varepsilon^{2}, \quad \mu \sim \varepsilon^{2}
$$

with covariant derivative $\partial_{\rho}$, pion fields $\pi(x)$, pion mass $m_{\pi}$, and chemical potential $\mu$. The corresponding systematic expansion of $\chi \mathrm{PT}$ is called epsilon expansion. To each order in $\varepsilon^{2}$ one can integrate out the spacetime dependence and obtain a finite-volume effective theory in terms of the constant pion mode. The order in $\varepsilon^{2}$ then translates into the order in $1 /\left(F^{2} \sqrt{V}\right)$. At leading order the finite-volume effective action is given by

$$
S_{\mathrm{eff}}^{\mathrm{LO}}=-\frac{1}{2} V \Sigma \operatorname{Tr}\left(M^{\dagger} U_{0}+U_{0}^{-1} M\right)-\frac{1}{2} V F^{2} \operatorname{Tr}\left(C U_{0}^{-1} C U_{0}\right)
$$

with constant pion mode

$$
U_{0}=\exp \left[i \pi_{0}\right], \quad \pi_{0}=\frac{1}{V} \int d^{4} x \pi(x),
$$

quark mass matrix $M=\operatorname{diag}\left(m_{1}, \ldots, m_{N_{f}}\right)$, and quark chemical potential matrix $C=\operatorname{diag}\left(\mu_{1}\right.$, $\left.\ldots, \mu_{N_{f}}\right)$, where $m_{f}$ is the quark mass and $i \mu_{f}$ is the imaginary chemical potential of quark flavor $f=1, \ldots, N_{f}$. We find that $S_{\text {eff }}^{\mathrm{LO}}$ is identical to the RMT action with nonzero chemical potential [6]. Note that the pion decay constant $F$ drops out for vanishing chemical potential. At next-to-leading order (NLO) in $\varepsilon^{2}$ the general form of Eq. (2.2) remains unchanged with $\Sigma \rightarrow \Sigma_{\text {eff }}^{\mathrm{NLO}}, F \rightarrow F_{\mathrm{eff}}^{\mathrm{NLO}}$ 
[9, 10,11]. In an actual lattice simulation we measure effective values $\Sigma_{\text {eff }}$ and $F_{\text {eff }}$, and we need to include finite-volume corrections to recover the infinite-volume values $\Sigma$ and $F$.

At NNLO and to leading order in the small chemical potential ${ }^{1}$ the effective action has the form [8]

$$
\begin{aligned}
S_{\text {eff }}^{\mathrm{NNLO}}= & -\frac{1}{2} V \Sigma_{\text {eff }}^{\mathrm{NNLO}} \operatorname{Tr}\left(M^{\dagger} U_{0}+U_{0}^{-1} M\right)-\frac{1}{2} V\left(F_{\mathrm{eff}}^{\mathrm{NNLO}}\right)^{2} \operatorname{Tr}\left(C U_{0}^{-1} C U_{0}\right) \\
& +\Upsilon_{1} \Sigma(V F)^{2} \operatorname{Tr}(C)\left[\operatorname{Tr}\left(U_{0}\left\{M^{\dagger}, C\right\}\right)+\operatorname{Tr}\left(U_{0}^{-1}\{C, M\}\right)\right] \\
& +\Upsilon_{2} \Sigma(V F)^{2} \operatorname{Tr}\left(\left\{M^{\dagger}, C\right\} U_{0} C+\{C, M\} C U_{0}^{-1}\right. \\
& \left.\quad+\left\{U_{0}, C\right\} U_{0}^{-1} C U_{0} M^{\dagger}+C U_{0}\left\{C, U_{0}^{-1}\right\} M U_{0}^{-1}\right) \\
& +\Upsilon_{3} \Sigma(V F)^{2} \operatorname{Tr}\left(U_{0}^{-1} C U_{0} C+C^{2}\right) \operatorname{Tr}\left(M U_{0}^{-1}+M^{\dagger} U_{0}\right) \\
& +\Upsilon_{4} \Sigma(V F)^{2} \operatorname{Tr}\left(U_{0}^{-1} C U_{0} C-C^{2}\right) \operatorname{Tr}\left(M U_{0}^{-1}+M^{\dagger} U_{0}\right) \\
& +\Upsilon_{5} \Sigma(V F)^{2} \operatorname{Tr}\left(\left[M^{\dagger}, C\right] U_{0} C+[C, M] C U_{0}^{-1}+\left[U_{0}, C\right] U_{0}^{-1} C U_{0} M^{\dagger}+C U_{0}\left[C, U_{0}^{-1}\right] M U_{0}^{-1}\right) \\
& +\Upsilon_{6}(V \Sigma)^{2}\left[\operatorname{Tr}\left(M U_{0}^{-1}+M^{\dagger} U_{0}\right)\right]^{2}+\Upsilon_{7}(V \Sigma)^{2}\left[\operatorname{Tr}\left(M U_{0}^{-1}-M^{\dagger} U_{0}\right)\right]^{2} \\
& +\Upsilon_{8}(V \Sigma)^{2}\left[\operatorname{Tr}\left(M U_{0}^{-1} M U_{0}^{-1}\right)+\operatorname{Tr}\left(M^{\dagger} U_{0} M^{\dagger} U_{0}\right)\right] \\
& +\mathscr{H}_{1} V F^{2} \operatorname{Tr}\left(C^{2}\right)+\mathscr{H}_{2}(V \Sigma)^{2} \operatorname{Tr}\left(M^{\dagger} M\right)+\mathscr{H}_{3} V F^{2}(\operatorname{Tr} C)^{2}
\end{aligned}
$$

with finite-volume effective coupling constants $\Upsilon_{i}$ and $\mathscr{H}_{i}$. The LECs $\Sigma$ and $F$ also receive further corrections, $\Sigma \rightarrow \sum_{\text {eff }}^{\mathrm{NNLO}}$ and $F \rightarrow F_{\text {eff }}^{\mathrm{NNLO}}$. The terms in Eq. (2.4) that were not present in Eq. (2.2) cannot be mapped to RMT. These terms are proportional to the $\Upsilon_{i}$ and $\mathscr{H}_{i}$. Therefore the magnitude of these coefficients determines the systematic deviations from RMT of, e.g., Dirac eigenvalue distributions. The coefficients $\mathscr{H}_{1}$ and $\mathscr{H}_{3}$ do not couple to $U_{0}$ or $M$ and are therefore irrelevant for Dirac eigenvalue distributions (which involve derivatives w.r.t. $M$ in the partially quenched theory). The coefficients $\Upsilon_{i}, \mathscr{H}_{2}, \Sigma_{\text {eff }}^{\mathrm{NNLO}}$, and $F_{\text {eff }}^{\mathrm{NNO}}$ depend on the NLO LECs of $\chi \mathrm{PT}$ and on the geometry of the spacetime box through finite-volume propagators. Explicit results are given in [8].

To be specific we discuss the following lattice geometries from now on,

$$
\begin{aligned}
& \left(a_{x}\right) \quad L_{0}=x L, \quad L_{1}=L_{2}=L_{3}=L, \\
& \left(b_{x}\right) \quad L_{3}=x L, \quad L_{0}=L_{1}=L_{2}=L,
\end{aligned}
$$

where $x \in\{1,3 / 2,2,3,4\}$, and $L_{i}$ is the extent of the spacetime box in direction $i$. In Fig. 1 we show the finite-volume corrections to $\Sigma$ and $F$ for the different geometries at NNLO for a set of parameters similar to the parameters of the JLQCD two-flavor epsilon-regime run [7]. We note that for the same asymmetry $x$, geometry $\left(b_{x}\right)$ leads to smaller finite-volume corrections to $F$ than geometry $\left(a_{x}\right)$. This was also observed in Ref. [11] at NLO. The finite-volume corrections to $\Sigma$ are invariant under $\left(a_{x}\right) \leftrightarrow\left(b_{x}\right)$. This is expected since $F$ is related to the chemical potential, which breaks the permutation symmetry of the four spacetime dimensions, while the same symmetry is intact for the $\Sigma$ term in $S_{\text {eff. }}$

We continue our discussion with the finite-volume effective coupling constants $\Upsilon_{i}$ and $\mathscr{H}_{2}$ that are responsible for the systematic deviations from RMT. It is an interesting observation that $\Upsilon_{1}, \Upsilon_{2}, \Upsilon_{3}$ do not depend on the NLO LECs of $\chi$ PT and depend on the geometry only through a

\footnotetext{
${ }^{1}$ There are also NNLO terms proportional to $V^{2} C^{4}$ that have been omitted in (2.4).
} 

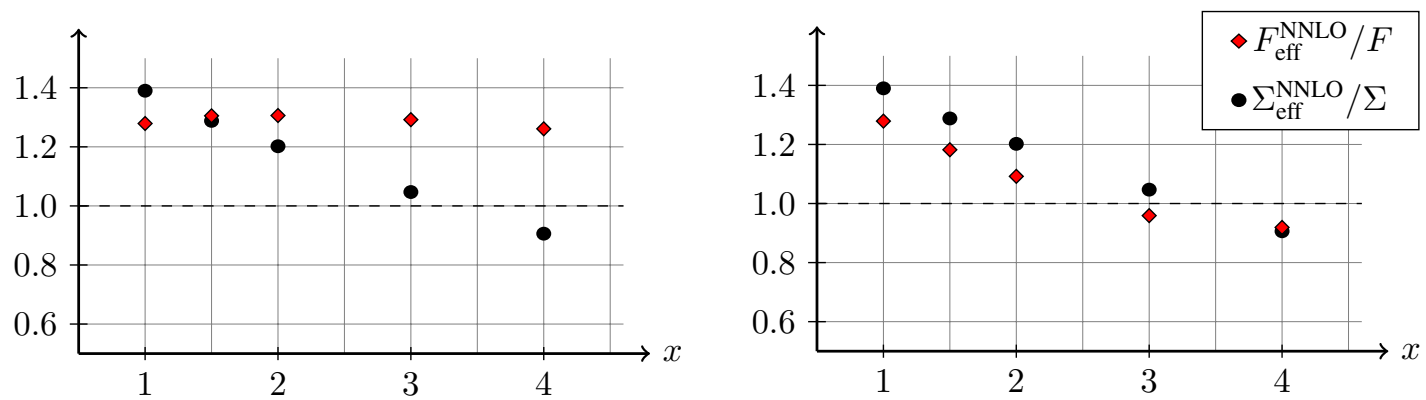

Figure 1: Finite-volume corrections to $\Sigma$ and $F$ for geometries $\left(a_{x}\right)$ on the left and $\left(b_{x}\right)$ on the right. The results are taken from Ref. [8] with parameters $F=90 \mathrm{MeV}, L=1.71 \mathrm{fm}$, and $m_{\pi}^{2} \sqrt{V}=1$.

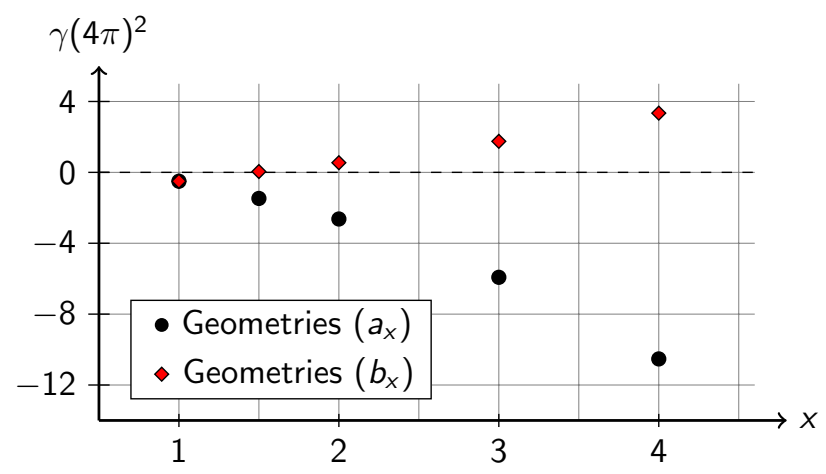

Figure 2: Geometry dependence of systematic deviations from RMT.

common coefficient $\gamma$, i.e.,

$$
\Upsilon_{1}, \Upsilon_{2}, \Upsilon_{3} \propto \gamma
$$

The coefficient $\gamma$ changes under $\left(a_{x}\right) \leftrightarrow\left(b_{x}\right)$, while $\Upsilon_{4}, \ldots, \Upsilon_{8}, \mathscr{H}_{2}$ are invariant under the same exchange [8]. We plot $\gamma$ for different geometries in Fig. 2 for the same set of parameters used in Fig. 1 . We note that the coefficient $\gamma$, and thus a part of the systematic deviations from RMT, can be reduced significantly by choosing the geometry $\left(b_{x}\right)$ instead of $\left(a_{x}\right)$ for the same value of the asymmetry $x$.

\section{Numerical results}

In this section we check the results of the previous section against the epsilon-regime run of JLQCD with two dynamical overlap fermions with mass $a m_{u}=a m_{d}=0.002\left(m_{\pi}^{2} \sqrt{V} \approx 1\right)$ and $32 \times 16^{3}$ lattice points at $a=0.107(3) \mathrm{fm}\left(V^{1 / 4} \approx 1.7 \mathrm{fm}\right)$ [7]. The sea quarks are at zero chemical potential, the valence quarks are at zero and nonzero imaginary chemical potential. In this way the existing configurations can be used to extract $\Sigma$ and $F$ with low numerical cost.

We first fit the distribution of the lowest-lying Dirac eigenvalue $P_{1}(\lambda)$ [12] in Fig. 3 in order to extract the finite-volume effective value

$$
a^{3} \Sigma_{\mathrm{eff}}=0.00208(2),
$$




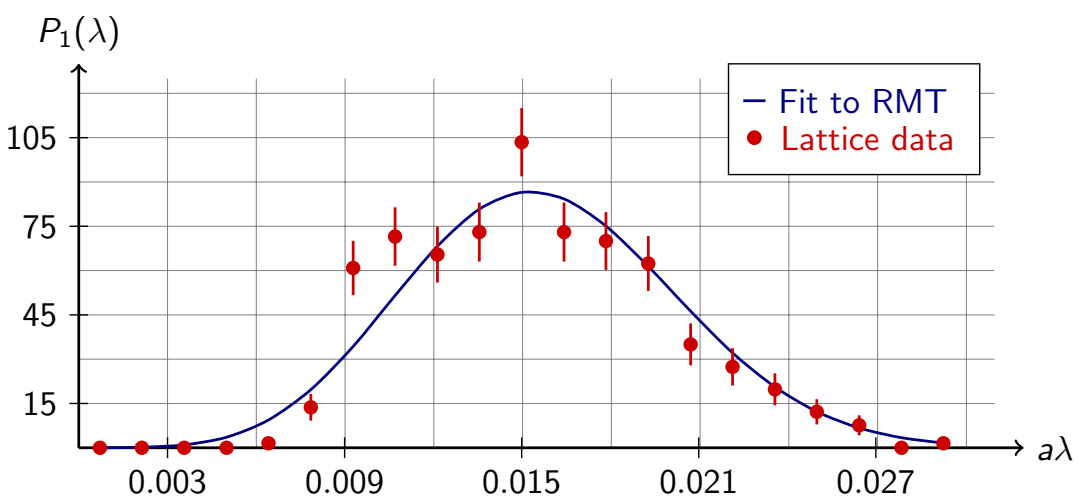

Figure 3: Fit to lowest-lying Dirac eigenvalue distribution $P_{1}(\lambda)$ with $\chi^{2} / \mathrm{dof}=2.9, a^{3} \Sigma_{\text {eff }}=0.00208(2)$.

where we only cite the statistical error. This value is compatible with $a^{3} \Sigma_{\text {eff }}=0.00212(6)$ obtained in Ref. [7] on the same configurations by a fit to the integrated Dirac eigenvalue distribution. Note, however, that there are significant systematic deviations from the RMT prediction in some regions of the Dirac eigenvalue $a \lambda$. Also note that both values for $\Sigma_{\text {eff }}$ are scheme dependent and that we give only the values for the lattice scheme here. Including finite-volume corrections at NLO gives an infinite-volume value $\Sigma=\Sigma_{\text {eff }} / 1.1454$ [8]. We use only NLO finite-volume corrections here and in the remainder of this paper since the NNLO finite-volume corrections and the systematic deviations from RMT are of the same order.

Next we fit the shift of the lowest-lying Dirac eigenvalue due to a small imaginary chemical potential $i \mu$ in order to extract $F$ as proposed in Ref. [5]. RMT predicts a Gaussian distribution with $\sigma^{2}=\mu^{2} F^{2} V$ for the distribution $P_{d}$ of the difference $d$ between the lowest Dirac eigenvalue at zero and at nonzero imaginary chemical potential $[5,6,13]$. In Fig. 4 we show the resulting fit for geometry $\left(a_{2}\right)$ with finite-volume effective value

$$
F_{\text {eff }}^{\left(a_{2}\right)}=67(5) \mathrm{MeV},
$$

where we only cite statistical errors. We note that the quality of the fit is rather bad $\left(\chi^{2} / \mathrm{dof}=4.2\right)$

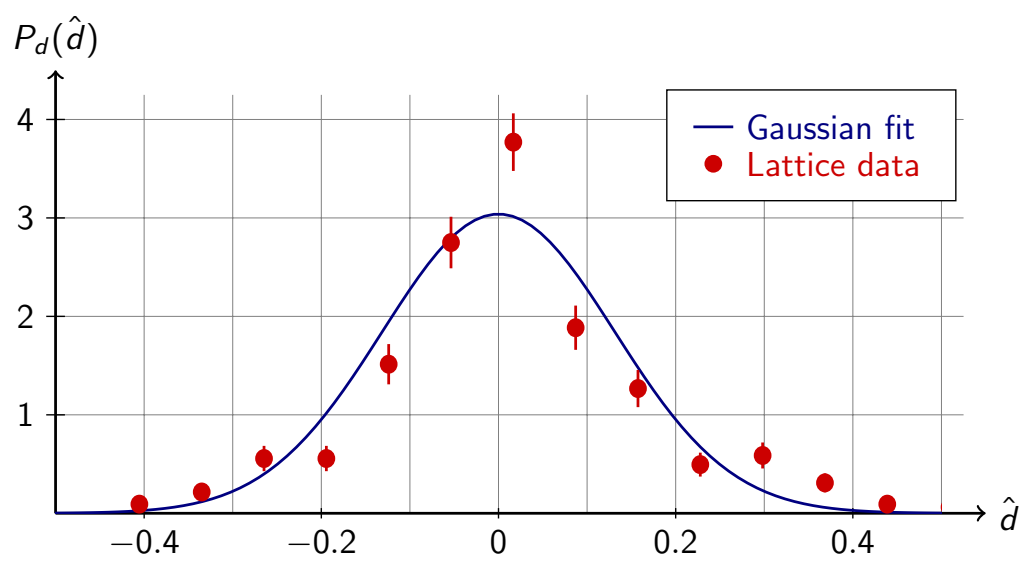

Figure 4: Fit to the distribution of Dirac eigenvalue shifts $P_{d}(\hat{d})$ due to imaginary chemical potential $a \mu=$ 0.01 with $\hat{d}=d \Sigma V$ in geometry $\left(a_{2}\right)$. The result is given by $F_{\text {eff }}=67(5) \mathrm{MeV}$ with $\chi^{2} / \mathrm{dof}=4.2$. 


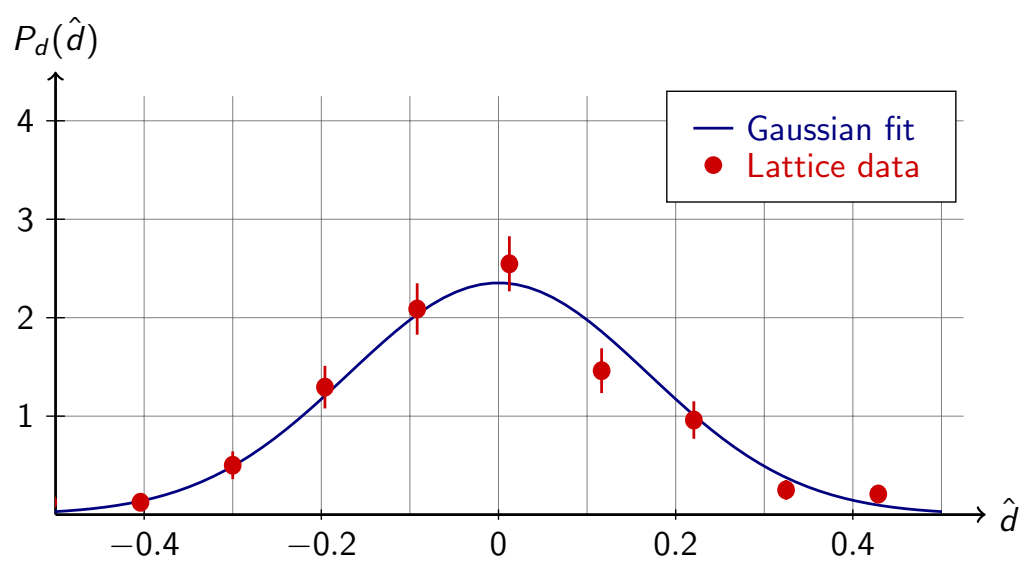

Figure 5: Fit to the distribution of Dirac eigenvalue shifts $P_{d}(\hat{d})$ due to imaginary chemical potential $a \mu=$ 0.01 with $\hat{d}=d \Sigma V$ in geometry $\left(b_{2}\right)$. The result is given by $F_{\text {eff }}=86(5) \mathrm{MeV}$ with $\chi^{2} / \mathrm{dof}=0.91$.

and that this value is not compatible with the result from a fit to meson correlators obtained on the same configurations [14], $F_{\text {meson }}=87.3(5.6) \mathrm{MeV}$. If we include finite-volume corrections at NLO we obtain the infinite-volume value

$$
F^{\left(a_{2}\right)}=51(4) \mathrm{MeV}
$$

so that the agreement is even worse. The bad $\chi^{2} / \mathrm{dof}=4.2$ suggests that the non-universal terms at NNLO, see Eq. (2.4) and the subsequent discussion, affect the distribution in a non-trivial manner.

From our discussion in the previous section we learned that we might be able to significantly reduce these systematic deviations from RMT by choosing lattice geometry $\left(b_{2}\right)$ instead of $\left(a_{2}\right)$. In practice this means that we should rotate the lattice by 90 degrees so that we have one large spatial dimension instead of a large temporal dimension. In Fig. 5 we show the resulting fit for geometry $\left(b_{2}\right)$ with $\operatorname{good} \chi^{2} /$ dof $=0.91$ and

$$
F_{\text {eff }}^{\left(b_{2}\right)}=86(5) \mathrm{MeV},
$$

where only statistical errors are given. Including finite-volume corrections at NLO this gives

$$
F^{\left(b_{2}\right)}=81(5) \mathrm{MeV},
$$

which agrees within errors with the result from the fit to meson correlators given above. Therefore we can confirm the analytical results of Ref. [8] in this specific example, and we can successfully use the method proposed in Ref. [5] to extract $F$ with competitive statistical errors.

\section{Conclusions}

We conclude that we can understand the geometry dependence of RMT-based methods to extract LECs from fits to Dirac eigenvalue distributions and that they provide a useful alternative if one chooses an optimized lattice geometry. 
Our final results for $\Sigma$ and $F$ obtained from the two-flavor epsilon-regime run of JLQCD are given by

$$
\Sigma^{\overline{\mathrm{MS}}}(2 \mathrm{GeV})=Z_{S}^{\overline{\mathrm{MS}}}(2 \mathrm{GeV}) \Sigma=(236(6) \mathrm{MeV})^{3}, \quad F=81(6) \mathrm{MeV},
$$

where both values include finite-volume corrections at $\mathrm{NLO}, \Sigma$ is the chiral condensate in the lattice scheme, and $Z_{S}^{\overline{\mathrm{MS}}}(2 \mathrm{GeV})=1.14(2)$ [7]. We give a combination of statistical errors and uncertainty in the lattice constant.

We note that within the framework of the finite-volume effective theory of Ref. [8] it is also possible to calculate Dirac eigenvalue distributions beyond RMT including the systematic deviations at NNLO in the epsilon expansion. Work in this direction is in progress.

\section{Acknowledgments}

This work was supported in part by BayEFG and the RIKEN FPR program (CL), the Grantin-Aid (No. 21674002) of the Japanese Ministry of Education (SH), and DFG and KEK (TW). The numerical calculations were carried out on the IBM System Blue Gene Solution at the High Energy Accelerator Research Organization under support of its Large Scale Simulation Program (No. 09/10-09).

\section{References}

[1] J. Gasser and H. Leutwyler, Phys. Lett. B188 (1987) 477.

[2] E. V. Shuryak and J. J. M. Verbaarschot, Nucl. Phys. A560 (1993) 306-320, [hep-th/ 9212088$].$

[3] J. J. M. Verbaarschot and T. Wettig, Ann. Rev. Nucl. Part. Sci. 50 (2000) 343-410, [hep-ph/0003017].

[4] F. Basile and G. Akemann, JHEP 12 (2007) 043, [arXiv: 0710 . 0376].

[5] P. H. Damgaard, U. M. Heller, K. Splittorff, and B. Svetitsky, Phys. Rev. D72 (2005) 091501, [hep-lat/0508029].

[6] G. Akemann, P. H. Damgaard, J. C. Osborn, and K. Splittorff, Nucl. Phys. B766 (2007) 34-67, [hep-th/0609059].

[7] JLQCD Collaboration, H. Fukaya et. al., Phys. Rev. Lett. 98 (2007) 172001, [hep-lat/ 0702003 ]; H. Fukaya et. al., Phys. Rev. D76 (2007) 054503, [arXiv: 0705.3322 ].

[8] C. Lehner, S. Hashimoto, and T. Wettig, JHEP 06 (2010) 028, [arXiv: 1004.5584 ].

[9] P. H. Damgaard, T. DeGrand, and H. Fukaya, JHEP 12 (2007) 060, [arXiv: 0711.0167 ].

[10] G. Akemann, F. Basile, and L. Lellouch, JHEP 12 (2008) 069, [arXiv: 0804 . 380 9].

[11] C. Lehner and T. Wettig, JHEP 11 (2009) 005, [arXiv: 0909 . 1489].

[12] T. Wilke, T. Guhr, and T. Wettig, Phys. Rev. D57 (1998) 6486-6495, [hep-th/9711057].

[13] P. H. Damgaard, U. M. Heller, K. Splittorff, B. Svetitsky, and D. Toublan, Phys. Rev. D73 (2006) 105016, [hep-th/0604054].

[14] H. Fukaya et. al., Phys. Rev. D77 (2008) 074503, [arXiv: 0711.4965$].$ 